\title{
A New Method for Semi-Supervised Segmentation of Satellite Images
}

\author{
Sara Sharifzadeh \\ Faculty of Engineering, Environment \\ and Computingdept. \\ Coventry University \\ Coventry, UK \\ ac8115@coventry.ac.uk
}

\author{
Sam Amiri \\ Faculty of Engineering, Environment \\ and Computingdept. \\ Coventry University \\ Coventry, UK \\ ad0246@coventry.ac.uk
}

\author{
Salman Abdi \\ School of Engineering \\ University of East Anglia \\ Norwich, UK \\ S.Abdi-Jalebi@uea.ac.uk
}

\begin{abstract}
Satellite image segmentation is an important topic in many domains. This paper introduces a novel semisupervised image segmentation method for satellite image segmentation. Unlike the semantic segmentation strategies, this method requires only limited labelled data from small local patches of satellite images. Due to the complexity and large number of land cover objects in satellite images, a fixed-size square window is used for feature extraction from local areas. Having the features from the labelled patches, the suitability of the window scale is found efficiently. Furthermore, the labeled features remove the need for iterative clustering for decision making about features. The labelled data also allows learning a subspace of transformed features for segmentation of water and green area based on simple thresholding. Comparison of the segmentation results using the proposed strategy compared to unsupervised techniques such as $k$-means clustering and Superpixel-based Fast Fuzzy C-Means Clustering (SFFCM) shows the superiority of the proposed strategy in terms of content-based segmentation.
\end{abstract}

Keywords-Satellite Image, unsupervised segmentation, semisupervised segmentation, formatting, feature clustering.

\section{INTRODUCTION}

Image segmentation is an important part of satellite image analysis. It finds application in many domains. Examples are urban data analysis and planning [1], digital agriculture [2,3], environmental studies and hazards analysis [4] and traffic and navigation [5].

One of the important applications of satellite image segmentation is identification of changes in landcover and cities over time. Identification of changes in urban areas can reveal information about environmental changes such as the green areas, the spread of residential and slum areas around cities and changes in water resources [6]. Such studies have great impact in relevant urban planning and social studies such air pollution, city planning for transportation, hazards and water resources, poverty control and citizens mental health issues.

Tracking changes in land cover based on satellite images requires segmentation of image objects and the land cover boundaries. Two main groups of image segmentation techniques are supervised and unsupervised strategies. In unsupervised approaches, no labels of the images are required. Since labelling satellite images can be quite time consuming, the unsupervised strategies are more suitable for satellite image analysis from this perspective. However, segmentation based on unsupervised strategies is not usually content-based and semantic. There are different unsupervised methods such as k-means clustering and Self Organising Maps (SOM) $[7,8]$, Multi Resolution Scaling [9], watershed transformation [10], hidden Markov random field (HMRF) [11], GraphCut [12], active contour model [13], fuzzy entropy [14], etc.

On the other hand, the supervised strategies require training labels such as deep learning segmentation strategies based on encoder-decoder structure [2] and convolutional neural network (CNN) [8]. Such methods accuracy is higher compared to the unsupervised strategies. They are also capable to semantically segment image objects in many cases. However, they usually require a large volume of data. Even, hundreds of labelled images are required when transfer learning methods are employed $[3,15]$.

One of the important groups of unsupervised image segmentation strategies are based on clustering. These fast strategies can be used for both high-resolution and lowresolution images. The image clustering algorithms can be categorised into three main groups [16]; (1) strategies that minimizing an objective function such as K-means and Fuzzy C-Mean (FCM) [16,17], (2) decompose a density function [18] (3) use graph theory $[19,20]$. The two main methods that fall into first group are k-means and FCM. k-means is known to be sensitive to initial cluster centres or membership [8]. On the other hand, FCM removes this limitation of K-means but at the cost of more computations.

Another important consideration on the use of clustering strategies is the scale and shape of the image local areas used for clustering. There are spatial information such as patterns and textures in local areas of digital images besides the colour or thermal information of individual pixels. Therefore, clustering at pixel level might influence the effectiveness and accuracy of clustering. That is especially important when semantics are important, which is the case for this study. Therefore, many research studies have addressed the use of clustering methods on local spatial information of images [21]. Neighbourhood information in local windows of fixed sizes and shapes [22] or adaptive neighbourhood shape and sizes have been developed at the expense of higher computational cost for neighbourhood analysis [23].

In the case of satellite images, due to the large size of images and complexity of patterns, the computational time is an important concern. Commercial softwares such as eCognition utilise universal and adaptable algorithms such Multi Resolution Scaling (MRS) [9] for segmentation. They allow users to select appropriate parameters for segmentation. This include the parameters for formation of local areas such as scale, sharpness and compactness and the type of features to extract from local areas for segmentation. They also allow to evaluate the results based on different supervised or unsupervised classification and clustering methods. The 
challenge will be when the scale and other factors should be found automatically.

This paper focuses on change detection in cities and their surrounding environments over time. Tracking changes requires content-based segmentation of satellite images. However, labelling large amount of satellite images for this aim is not practical. Therefore, to compromise between accurate semantic segmentation using supervised methods and unsupervised segmentation, a semi-supervised strategy is proposed. Then the main contributions of the paper are:

(1) Proposing a novel semi-supervised segmentation method for satellite images. The method uses both individual pixel information such as colour and Infra-Red (IR) thermal range as well as local spatial texture information extracted from local areas of the satellite images as features. Using the limited labelled data set, helps to (a) use their corresponding features for decision making. This is performed based on similarity of the extracted features from local areas to the labelled areas. This remove the need for cluster centres as in K-means clustering for decision making (b) The labelled data also helps to use fast fixed-size and shape windows for feature extraction from local neighbourhoods. That is due to the fact that having the limited set of labelled areas features, the suitability of segmentation results at different scales can be evaluated more effectively. Unlike the unsupervised strategies the automated scale selection is performed using both contentbased segmentation results and homogeneity of the segmented areas. Then is achieved based on calculating a similarity measure between the labelled features and the acquired local features at a given fixed-size and shape windows. This can be performed at different scales and the best scale (window size) can be identified. The evaluation is based on both homogeneity of the segments and their similarity to the labelled areas.

(2) Furthermore, based on simple thresholding, segmentation of two important class of water and green area is achieved. That is obtained by utilisation of the limited labelled samples to learn a subspace of features and transforming images into the new subspace. The subspace is learnt using colour and thermal bands pixels. The generalised eigen decomposition of within-class and between-class covariance matrices based on Rayleigh quotient [24,25] is employed. The resulting eigen vectors allow transforming the visible and thermal IR bands into a reduced subspace. In the new subspace, the distance of the green and water areas with respect to each other and the rest of classes is increased, allowing simple thresholding for segmentation.

(3) The suggested method results are compared with two unsupervised strategies based on K-means clustering and the Superpixel-based Fast FCM clustering algorithm (SFFCM) [2018].

The rest of the paper is organised as follows. Section two is about materials and methods. Data description and description of the previous methods and the proposed strategy is conducted in this section. Section three presents the results. Finally a discussion and conclusion is presented in section four.

\section{MATERIALS AND METHODS}

This section describes the satellite images used in this work. In addition, the techniques used for segmentation of different areas of the images are described.

\section{A. Data Description}

In this paper, two satellite images of Landsat4-5 of Tehran are considered. The images were recorded in August 1985 and July 2011 respectively. In each case, RGB images as well as thermal IR band (1040-1250 nm) are used for analysis. Only these two bands data were available for this study. The thermal IR band represents the thermal and soil moisture condition. The resolution of landsat 4-5 images are $30 \mathrm{~m}$. Error! Reference source not found.. illustrates the two images. As can be seen, the images are cloud-free that makes them suitable for segmentation.

For segmentation of different areas in the images, first the most prominent classes of objects are identified. This include water, residential, mountain, green, farm, road and land area. Such area can be observed in both images.
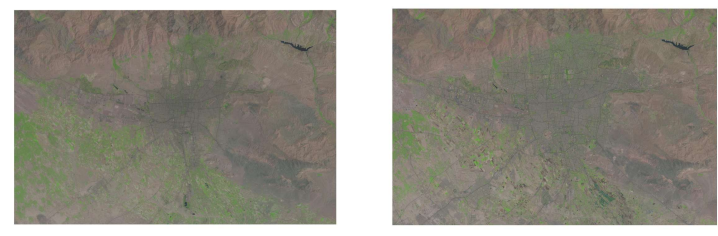

Fig. 1. RGB images of Tehran(left) August 1985, (right) July 2011.

\section{B. K-means Clustering for Segmentation}

$\mathrm{K}$-means clustering calculates the distance of all pixel grey values from initial randomly defined cluster centroids. Based on the distance, the pixels are assigned to the clusters. Then, the cluster centroids are updated and the same steps are repeated until there are no further changes in the cluster centres [k-means].

\section{C. $\quad$ Superpixel-based Fast Fuzzy C-Means Clustering}

The SFFCM algorithm uses a multiscale morphological gradient reconstruction (MMGR) operation to generate super pixels in an image. The super pixels have accurate adaptive and irregular boundaries. The local spatial neighbourhood allows segmentation using colour information. The original colour image is used to calculate the histogram of colour sin each region. Finally, the fast FCM is applied on the histogram parameter of the superpixel image to obtain the final segmentation result. For further information, we refer to [SFFCM]

\section{The Proposed Semi-Supervised Segmentation Method}

In order to provide limited labelled samples, from different areas of the satellite images, some local patches of data are collected.

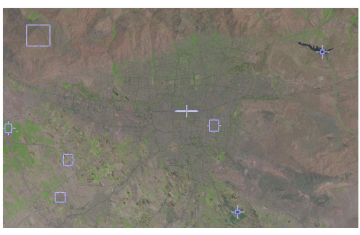

Fig. 2. Illustration of the limitted local patches of image in from different classes of water, mountain, residential, farm, green, land and road area.

These small patches are used to extract both individual pixels colour and thermal IR information and also the spatial texture 
information from the local areas. Therefore, the average of RGB and IR band grey values is computed for all patches, giving 4 features per patch. In addition, the 2-dimensional Discrete Cosine Transform (DCT) is applied to extract the texture information. Since the local patches are homogenous in structure, only the low frequency DCT coefficients describing the main patterns of the local patches are considered. For this aim the $3 \times 3$ DCT coefficients from the top-left corner of the 2D DCT map are extracted, yielding 9 texture features. Therefore, a total of 13 features are computed for each class.

The next step is to form local square windows for the total satellite images. Then, similar features can be extracted for each of them. The features can be used to assign each local area of the image (under the window) into one of the classes. The decision for assignment can be based on similarity to the features of labelled patches.

The main challenge for formation of the local window areas is the choice of scale or size of window. With small window, the spatial information will be lost. On the other hand, a big window sizes will result into under-segmentation that ruin the homogeneity of the local area and dissimilarity to the features of all labelled patches. Therefore, the segmentation is performed for different window sizes and based on the joint measures of (1) under-segmentation that causes heterogeneity of the segments and (2) the local segments similarity to the labelled patches, the optimum window size is chosen.

\section{E. Rayleigh Qutient for Feature Transformation}

Rayleigh quotient is a feature transformation method that allows to increase the distance of the samples of different clusters. This is performed by learning eigen values for transforming data into a new space. For this aim the three RGB layers as well as the thermal RGB band data are used, yielding a total of 4-dimensional variable space. First, the mean and covariance of the limited labelled samples in each of the 7 classes is computed first $\mu_{i}$ and $\sum_{i}, i=1,2, \ldots, 7$. Then, the two within-class covariance $S_{w}=\sum_{i=1}^{7} \sum_{i}$ and between-class covariances $S_{\boldsymbol{B}}=\sum_{i=1}^{7} n_{i}\left(\mu_{i}-\mu_{t}\right)\left(\mu_{i}-\mu_{t}\right)^{2}$ are computed. $n_{i}$ is the number of labelled samples in the $i^{\text {th }}$ class and $\mu_{t}$ is the total mean of all of the class's features. Next, a generalised eigen decomposition is performed to maximize the Rayleigh Quotient objective function:

$$
R\left(S_{w}, C, w\right)=\max _{w} \frac{w^{T} S_{\boldsymbol{B}} w}{w^{T} S_{\boldsymbol{w}} w}
$$

The resulting Eigen vector $(w)$ corresponds to the largest Eigenvalue. It is possible to compute several eigen vectors equal to the number of variables e.g. 4 in this work. By transforming the original samples into a new orthogonal space using the eigen vectors, it can be observed that the geometrical distribution of different clusters data and their distances change. That allows forming images that different clusters pixel values can be easily distinguished from other clusters so that they can be segmented easily by thresholding. That is due to the fact that Rayleigh quotient objective function, tries to minimize the distance between the samples in each cluster based on $\left(S_{w}\right)$, while their distance to the samples of the other clusters are maximized $\left(S_{\boldsymbol{B}}\right)$. Then, the results should be a reduction in the overlap between some clusters data.
In this work, the appropriate eigen vectors for transforming data are identified based on visualisation of the transformed labelled data. Then, the selected eigen vector is used for transforming the whole satellite image pixels $X$ into a new space $Z=X w$, where is the vectorised satellite image of four layers $X_{N \times 4}$ and $\mathrm{N}$ is the number of all pixels in each satellite image layer and $w_{4 \times 1}$ is the selected eigen vector.

\section{F. Evaluation of Segmentation}

The average area-weighted variance (WV) measures [26] is used for intra-segment homogeneity. The higher values shows more under-segmentation and lower values is showing less under segmentation. For a given image band, the variance of pixels in each segment is computed and weighted by the segments area. This is computed over all segments and averaged. The result is also divided by the sum of the areas of all segments. This is shown in Error! Reference source not found., where $n$ is the number of segments, $a_{i}$ represents the area of a segment and $v_{i}$ is the variance within a segment.

$$
W V=\frac{\sum_{i=1}^{n} a_{i} v_{i}}{\sum_{i=1}^{n} a_{i}}
$$

Another criterion that is used for the proposed semisupervised frame work is the dissimilarity of the segmented area to the labelled patches. This shown in (2), where $C$ is the total number of clusters and $n_{i}$ is the total number of segments clustered into $i^{\text {th }}$ cluster and $f_{j}$ is the 13 length feature vectors of the $j^{\text {th }}$ segment of $i^{\text {th }}$ cluster and $f_{i}$ is the feature vector of cluster $i$.

$$
D=\frac{\sum_{i=1}^{C} \sum_{j=1}^{n_{i}} \sqrt{\left(f_{j}-f_{i}\right)^{2}}}{\sum_{i=1}^{C} n_{i}}
$$

This measure is used to find the optimum local neighbourhood window size for segmentation.

\section{EXPERIMENTAL RESULTS}

\section{A. Kmeans Clustering Segmentation Result}

The result of applying K-means clustering on RGB images for the seven classes of water, residential, mountain, green, farm, road and land area are shown in Error! Reference source not found. for the two years. There is not undersegmentation in the visualised results. The WV measures shown in TABLE I. are also not high. In terms of identified clusters, some classes are mixed such as water area that in 1985 image has the same green colour labels as parts of the streets and residential areas. In the case of 2011 image, the water area has similar cyan colour label as some of the green and mountain areas

\section{B. SFFCM Clustering Result}

The result of segmentation of RGB images using SFFCM is visualized in Fig. 4. The labels of the seven clusters show confusion in some areas. The most prominent is the water area that is clusters with parts of residential areas in both cases. There is less over-segmentation in k-means results compared to SFFCM. This is shown also in TABLE I. 

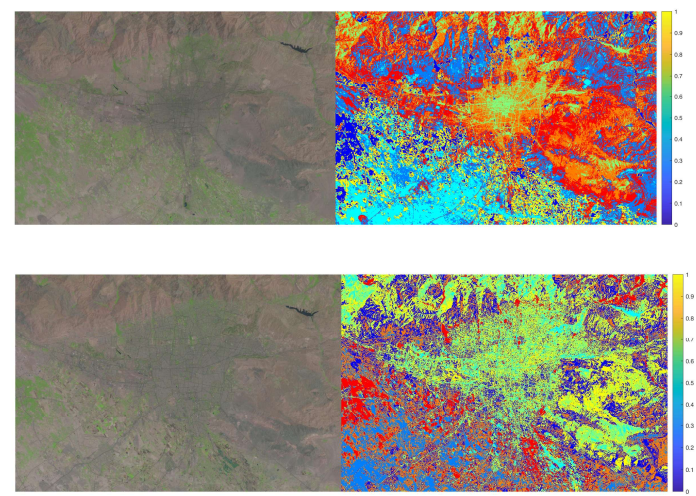

Fig. 3. Segmentation results based on K-means clustering of pixels using RGB images (top) 1985 (bottom) 2011.
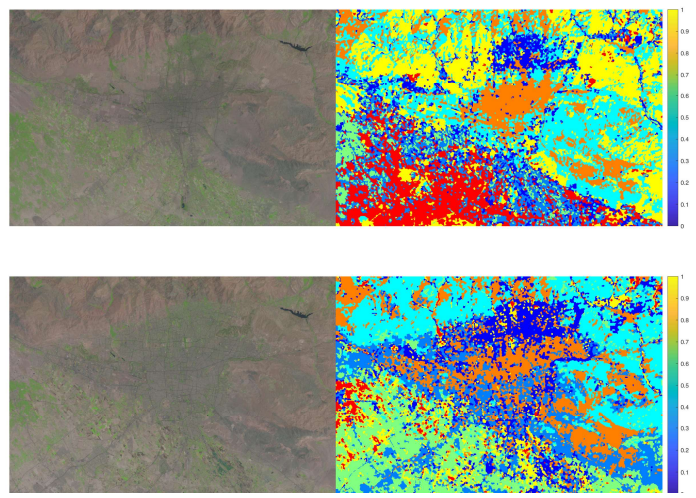

Fig. 4. Segmentation results based on SFFCM clustering of pixels using RGB images (top)1985 (bottom) 2011.

\section{The Proposed Semi-Supervised Strategy Result}

The result of the proposed semi-supervised strategy is presented in Fig. 5. As can be seen, the over-segmentation issue improved. This is also shown in TABLE I. Since both

RGB and thermal IR band is used, the WV measure is calculated by averaging the results over four bands. In terms of the segmented content, compared to other techniques, there are some improvements. For example, the water area is not confused with other clusters. In addition, the green area in the southern part of Tehran in 1985 images are better segmented using the proposed method.

The scale for the size of neighborhood window is chosen as 12. The reason for selection of this scale is based on the calculation of the dissimilarity $D$ and $W V$ measures for different window size ranges. They are shown in TABLE II. As can be seen the lowest average dissimilarity is obtained for the window size 12 . That means, the most similarity in between the features of the labelled clusters and their corresponding assigned segments is achieved for this local neighborhood window size. In addition, the $W V$ as the measure of under-segmentation rises in higher scales, while it gives over-segmentation in the smaller scale than 12 TABLE I. shows the average WV measures for the three segmentation methods.
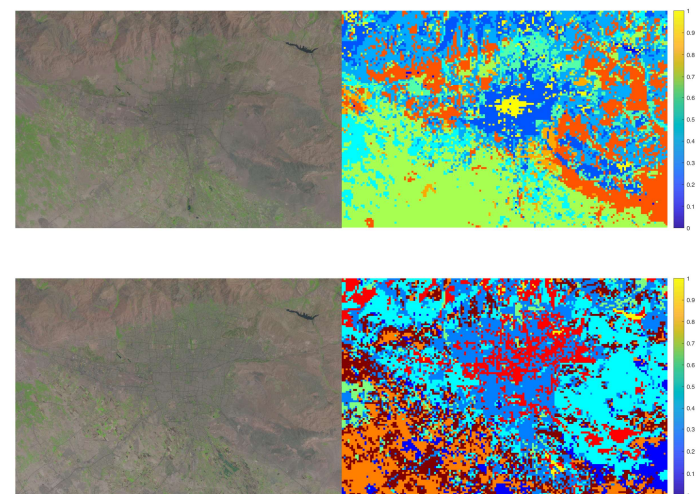

Fig. 5. Segmentation results based on the proposed semi-supervised strategy (top)1985 (bottom) 2011.

TABLE I. THE DISSIMILARITY MEASURE $D$ FOR FOUR WV MEASURE FOR SEGMENTATION

\begin{tabular}{|l|c|c|c|}
\hline \multirow{2}{*}{ Year } & \multicolumn{3}{|c|}{ WV Measure } \\
\cline { 2 - 4 } & K-means & SFFCM & Proposed Method \\
\hline 1985 & 12.9943 & 33.2944 & 54.7048 \\
\hline 2011 & 12.2434 & 46.4626 & 54.6536 \\
\hline
\end{tabular}

TABLE II. D AND WV MEASURES FOR THE PROPOSED

\begin{tabular}{|c|c|c|c|c|c|c|c|c|}
\hline $\begin{array}{c}\text { Window } \\
\text { size }\end{array}$ & \multicolumn{2}{|c|}{6} & \multicolumn{2}{|c|}{12} & \multicolumn{2}{c|}{20} & \multicolumn{2}{c|}{30} \\
\hline Year & $\boldsymbol{D}$ & $\boldsymbol{W V}$ & $\boldsymbol{D}$ & $\boldsymbol{W} \boldsymbol{V}$ & $\boldsymbol{D}$ & $\begin{array}{c}\boldsymbol{V} \\
\boldsymbol{V}\end{array}$ & $\boldsymbol{D}$ & $\boldsymbol{W} \boldsymbol{V}$ \\
& 18. & 41.2 & 13. & 54.6 & 13. & 64. & 13.9 & 71.8 \\
1985 & 17 & 9 & 28 & 7 & 56 & 87 & 4 & 5 \\
\hline \multirow{2}{*}{2011} & 15. & 41.2 & 12. & 54.6 & 13. & 63. & 13.6 & 70.4 \\
& 66 & 2 & 95 & 3 & 29 & 88 & 7 & 8 \\
\hline
\end{tabular}

\section{Change Detection Based on Segmentation Results}

Using the segmentation results of the proposed semisupervised framework, the percentage of areas belonging to each cluster is computed. Depending on the nature of cluster type, e.g. farm or residential area, their variations between 1985 and 2011 are important for interpretation. Therefore, the percentage of changes in those clusters that can be interpretable is visualized in Fig. 6. As can be seen, based on the segmentation results the green and farm areas in Tehran and its nearby area have been decreased, while the residential areas and water area have increased.

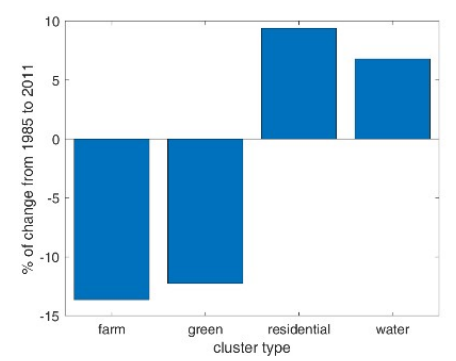

Fig. 6. Illustration of the percentatge of cahnges for interpretable clusters in terms of changes between 1985 and 2011 


\section{E. Segmentation Results using Rayleigh Qutient}

The application of Rayleigh Quotient on the labelled samples in four layers of RGB and IR thermal band, resulted in an increase mainly in the distance of water and green area samples as shown in Fig. 7 (left). As can be seen in the 3D plot, along the horizontal axis labeled (f2) that corresponds to the second eigen vector, the red cluster of water samples and black cluster of green area are well separated. Using this eigen vector the four layers of the satellite image is transferred into the new space. That is shown in Fig. 7 (right). The highest gray values as shown in the color bar corresponds to water area while the lowest gray values correspond to the green area. Then, simple thresholding is applied to segment the water and green area in this case.

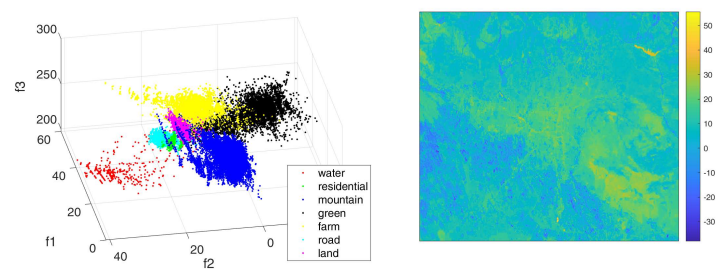

Fig. 7. (left) The $3 \mathrm{D}$ visualisation of the transferred labelled samples of different clustres using the three different eigen vectors. (right) the transferred satellite image of year 1985 using the second eigen vector.
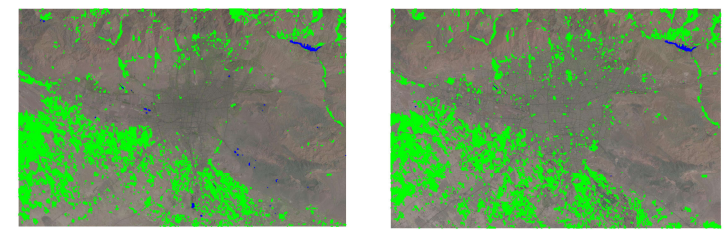

Fig. 8. The segmentation of water and green area by thresholding the transformed satellite images based on the eigen vectos found by Rayleigh Qutient, (left) August 1985 (right) July 2011

\section{DISCUSSION AND CONCLUSION}

The segmentation results obtained based on the three segmentation strategies showed that using the individual pixel color information using the k-means clustering, results into over segmentation as the local spatial information are not considered. On the other hand, the use of SFFCM algorithm on RGB images reduced the over segmentation. The proposed semi-supervised segmentation strategy improved further the content-based segmentation and reduced the confusion between clusters. The simplified fixed-size neighborhood and the extracted features from the limited labeled area, reduced the computation time compared to the SFFCM and remove the need for K-means iterations and sensitivity to the initial clusters.

The segmentation results based on thresholding of the transformed satellite images using the learnt subspaces based on Rayleigh quotient can be used for improving the results obtained based on the proposed semi-supervised strategy.

Besides the DCT analysis for quantification of the local spatial patterns, there are other texture analysis techniques that can be employed in future works. In addition, the proposed strategy can be applied on adaptive neighborhood and local boundaries in future works.

\section{CONCLUSION}

In this paper, a semi-supervised image segmentation strategy is proposed for segmentation of satellite images. Unlike the semantic segmentation methods, the proposed strategy requires limited labelled samples from local areas of the image. These samples allow the method to guide the decision process for the clustering of the local features acquired from satellite images. They also remove the sensitivity concerns of the k-means clustering methods to the initial cluster centers. The proposed strategy utilizes both individual pixels RGB and thermal information and the spatial information acquired from local neighborhood. Having the limited labelled features from different clusters, the decision about the size of the windows of neighboring area is automated and regular shape fixed size windows are used. By alternating the size of square shape windows, the result of segmentation is evaluated in terms of both similarity to the labelled data and over- or under-segmentation. That allows utilizing fast fixedshape and -size windows, while maintaining both contentbased similarity and homogeneity of the segments. Furthermore, features are transformed into sub-spaces based on Rayleigh quotient that allowed simple thresholding for segmenting water and green areas. Finally, using the segmentation results, changes in areas of the urban environment over time that can be interpreted are identified.

\section{REFERENCES}

[1] D. Kohli, R. Sliuzas, A. Stein, "Urban slum detection using texture and spatial metrics derived from satellite imagery", Journal of Spatial Science, Vol. 61(2), PP. 405-426, 2016.

[2] S. Sharifzadeh, J. Tata, H. Sharifzadeh, B. Tan, "Farm Area Segmentation in Satellite Images Using DeepLabv3+ Neural Networks", Springer Communications in Computer and Information Science (CCIS), Vol. 1255, 2020.

[3] S. Sharifzadeh, J. Tata, B. Tan "Farm Detection Based on Deep Convolutional Neural Nets and Semi-Supervised Green Texture Detection using VIS-NIR Satellite Image", 8th International Conference on Data Science, Technology and Applications, Czech, 2019.

[4] S. Voigt, T. Kemper, T. Riedlinger, R. Kiefl, K. Scholte and H. Mehl, "Satellite Image Analysis for Disaster and Crisis-Management Support," in IEEE Transactions on Geoscience and Remote Sensing, vol. 45, no. 6, pp. 1520-1528, June 2007.

[5] W. Wang, N. Yang, Y. Zhang, F. Wang, T. Cao, P. Eklund, "A review of road extraction from remote sensing images", Journal of Traffic and Transportation Engineering (English Edition), Vol. 3 (3), pp. 271-282, 2016.

[6] L. Martinez, V. Pala, R. Arbiol, L. Pineda and M. Joaniquet, "Urban Change Detection on Satellite Images Series. Application to Catalunya Area," 2007 Urban Remote Sensing Joint Event, Paris, 2007, pp. 1-5, doi: 10.1109/URS.2007.371834..

[7] M. Awad, "An Unsupervised Artificial Neural Network Method for Satellite Image Segmentation”, Int. Arab J. Inf. Technol., Vol. 7(2), pp. 199-205, 2010.

[8] D. Arthur, S. Vassilvitskii, "k-means++: The Advantages of Careful Seeding." SODA '07: Proceedings of the Eighteenth Annual ACMSIAM Symposium on Discrete Algorithms, pp. 1027-1035, New Orleans, LA, January 2007. [k-means]

[9] M. Baatz and A. Schäpe, "Multiresolution Segmentation : an optimization approach for high quality multi-scale image segmentation", In: Strobl, J., Blaschke, T. and Griesbner, G., Eds., Angewandte Geographische Informations-Verarbeitung, XII, Wichmann Verlag, Karlsruhe, Germany, 2000. 
[10] M. Bai and R. Urtasun, "Deep watershed transform for instance segmentation," in Proc. IEEE Conf. Comput. Vis. Pattern Recognit. (CVPR), Honolulu, HI, 2017, pp. 2858-2866.

[11] M. Pereyra and S. McLaughlin, "Fast unsupervised bayesian image segmentation with adaptive spatial regularisation," IEEE Trans. Image Process., vol. 26, no. 6, pp. 2577-2587, Jun. 2017.

[12] J. Ma, S. Li, H. Qin and A. Hao, "Unsupervised multi-class cosegmentation via joint-cut over L1-manifold hyper-graph of discriminative image regions," IEEE Trans. Image Process., vol. 26, no. 3, pp. 1216-1230, Mar. 2017.

[13] M. Gong, H. Li, X. Zhang, Q. Zhao and B. Wang, "Nonparametric statistical active contour based on inclusion degree of fuzzy sets," IEEE Trans. Fuzzy Syst., vol. 24, no. 5, pp. 1176-1192, Oct. 2016.

[14] S. Yin, Y. Qian and M. Gong, "Unsupervised hierarchical image segmentation through fuzzy entropy maximization," Pattern Recognit., vol. 68, pp. 245-259, Aug. 2017.

[15] A. Krizhevsky, I. Sutskever and G. E. Hinton, "Imagenet classification with deep convolutional neural networks," in Proc. Adv. Neural Inf. Proc. Syst. (NIPS), New York, NY, pp. 1097-1105, 2012.

[16] T. Lei, X. Jia, Y. Zhang, Sh. Liu, H.Meng and A. K. Nandi, "Superpixel-based Fast Fuzzy C-Means Clustering for Color Image Segmentation", IEEE Transactions on Fuzzy Systems, vol. 27, no. 9, pp. 1753-1766, 2019, doi: 10.1109/TFUZZ.2018.2889018.

[17] N. R. Pal and J. C. Bezdek, "On cluster validity for the fuzzy c-means model,” IEEE Trans. Fuzzy Syst., vol. 3, no. 3, pp. 370-379, Aug. 1995.

[18] D. Comaniciu and P. Meer, "Mean shift: A robust approach toward feature space analysis," IEEE Trans. Pattern Anal. Mach. Intell., vol. 24, no. 5, pp. 603-619, May 2002.
[19] A. Y. Ng, M. I. Jordan and Y. Weiss, “On spectral clustering: Analysis and an algorithm," in Proc. Adv. Neural Inf. Proc. Syst. (NIPS), Vancouver, British Columbia, pp. 849-856, 2001.

[20] Y.Boykov, G. Funka-Lea, "Graph Cuts and Efficient N-D Image Segmentation”, Int J Comput Vision 70, 109-131, 2006.

[21] Y. Zhang, X. Bai, R. Fan and Z. Wang, "Deviation-sparse fuzzy cmeans with neighbor information constraint," IEEE Trans. Fuzzy Syst., 2018. Doi: 10.1109/TFUZZ.2018.2883033.

[22] K. H. Memon and D. H. Lee, "Generalised fuzzy c-means clustering algorithm with local information," IET Image Process., vol. 11, no. 1, pp. 1-12, Jan. 2017.

[23] G. Liu, Y. Zhang and A.Wang, "Incorporating adaptive local information into fuzzy clustering for image segmentation," IEEE Trans. Image Process., vol. 24, no. 11, pp. 3990-4000, Nov. 2015.

[24] B. Parlett, "The symmetric eigenvalue problem", Society for industrial and applied mathematics, Prentice-Hall, 1998.

[25] Sh. Yu, L. Tranchevent, B.M.Y. Moor, "Kernel-based data fusion for machine learning: methods and applications in bioinformatics and text mining", Springer, 2011.

[26] G.M. Espindola, G. Camara, I.A. Reis, L.S. Bins, A.M. Monteiro, "Parameter selection for region-growing image segmentation algorithms using spatial autocorrelation", Int. J. Remote Sens. Vol. 27, pp. 3035-3040, 2006. [33] 